\title{
Analysis of the effect of an obstacle on the wind energy potential
}

\author{
N. Amahjour ${ }^{1}$, and A. Khamlichi ${ }^{2}$ \\ ${ }^{1}$ Departement of Physics, Faculty of Sciences, University Abdelmalek Essaadi, Tetouan 93030, Morocco \\ ${ }^{2}$ Departement TITM, National School of Applied Sciences, University Abdelmalek Essaadi, Tetouan 93030, Morocco
}

\begin{abstract}
Assessment of the wind energy potential for a given site requires a primitive evaluation of wind speed for a specified period of time. Wind speed distribution depends on the type of air flow in the atmospheric boundary layer and varies as function of the altitude and the site topography. It is also affected by the presence of obstacles, the curvature radius of obstacle, the roughness of the ground and temperature. In this study, circular motion of the atmosphere that is induced by the existence of an obstacle having the form of a hill is investigated by using a 2D model. The hill profile was approximated by a half-ellipse curve. Computational fluid dynamics (CFD) was performed for the turbulent air flow modelled by the Navier-Stokes equations coupled with the K- $\varepsilon$ turbulence model. To solve the equations, COMSOL software that is based on the finite element method was used. The obtained results were compared to those of a flat ground without obstacles. A noticeable modification of the available wind energy resulted for a wind turbine which is placed downwards of a hill like obstacle.
\end{abstract}

\section{Introduction}

The increasing concerns with environmental and economical issues are driving the search for more sustainable electrical sources. Wind energy along with solar energy, biomass and wave energy are all possible solutions for environmentally friendly energy production. Wind energy has shown however to be the most prominent renewable energy source for regions where wind speed fulfils ideal characteristics in terms of magnitude and stability. In these last decades, the installation of a great number of wind turbine generators has effectively reduced environmental pollution, fossil fuel consumption, and the costs of overall electricity generation. Although wind is only an intermittent source of energy, it represents a reliable energy resource from a long-term energy policy viewpoint. At a specific wind farm, the available electricity generated by a wind power generation system depends on the mean wind speed, the direction of wind and the standard deviation of wind speed. These quantities vary also as function of the altitude and topographic details of the wind turbine location of installation [1].

Evaluating the amount of the wind energy potential for a given site requires an earlier estimation of wind velocity that is susceptible to be captured by the wind turbine rotor. This needs to be performed during a sufficiently large period of time based on accurate height distribution of wind speeds on that location. Wind velocity distribution is transient in nature and depends on the type of air flow in the atmospheric boundary layer. It varies as function of the altitude and the site topography. It is also affected by the presence of obstacles, the curvature radius of obstacle, the roughness of the ground and temperature.

The air flowing across an obstacle having the form of a solid wall surface will stick to that surface. This is due to adherence forces and viscosity of the fluid which produce the non-slip condition at the wall. As a result a boundary layer is formed near the obstacle where the velocity varies from zero at the wall to a finite value at some sufficient distance from that wall. There are actually many procedures that can be used to study the boundary layer characteristics in terms of skin-friction, dissipation, turbulence and loads that can be generated.

The pioneering work is attributed to Prandtl [2]. He introduced an important assumption that enables to reduce the Navier-Stokes equations. Using the concept of dimensional analysis he has determined the similarity parameters governing the laminar boundary layer $[3,4]$. Computational Fluid Dynamics (CFD) [5] is a modern approach that is used to solve numerically the turbulent air flow equations around complex obstacles. CFD is based on the Navier-Stokes equations which are coupled to a turbulence closure model such the k- $\varepsilon$ model [6]. The main advantage of CFD is that it is a very compelling, non-intrusive, virtual modeling technique with powerful visualization capabilities. Engineers can evaluate the 
performance of a wide range of system configurations on the computer without the time, expense, and disruption required to make actual changes onsite. This technique has widely been applied to various engineering applications such as automobile, aircraft design and civil engineering, where CFD data was utilized to evaluate various parameters in order to meet the required design criteria.

In this work, simulation of the turbulent flow occurring around an obstacle that is present on a flat ground is performed. A two-dimensional model describing air circulations that are induced by the presence of the obstacle chosen to have the form of an ellipse is developed. Appropriate boundary layer conditions are applied. The equations are solved by using the finite element based COMSOL software package [7].

Like in every finite element calcualtion, the precision of the obtained solution has to be assessed. This depends in the actual analysis on the location of the boundary fixing the domain of the air and also the mesh used. Convergence of the finite element model is studied here asymptotically by modifying the frontier of the domain and the mesh size until a stationnary solution is obtained. The results are compared then with those corresponding to the case of a flat ground without any obstacle. This is done at the aim of catching the spatial heterogeneities of the air flow which is due to obstacles. The results are useful in particular for re-estimating the profiles of wind speed as affected by flow turbulence around complex geometries, which in their turn can be used to accurately predict wind energy potential for a given site location of the wind turbine.

\section{Geometry and mathematical formulation}

The geometry of the problem consists of a horizontal ground with a hill form obstacle that can be assimilated to an ellipse. The domain of fluid to be modeled is part of the problem and has to be determined through an iterative procedure which assures a stationary solution for a frontier located at sufficiently large distance from the obstacle.

The CFD modeling is implemented by using the COMSOL package software. The air flow near a flat plate with an obstacle is typically turbulent. The partial differential equations that describe the stationary fluid motion are the well known Navier-Stokes equations which write:

$$
\begin{aligned}
& \rho \nabla .(U)=0 \\
& \rho(U . \nabla) U=\nabla\left[-P+\mu\left(\nabla U+(\nabla U)^{t}\right)\right]+F
\end{aligned}
$$

where $U$ is the velocity vector, $\rho$ the density, $\mu$ the dynamic viscosity, $P$ the pressure, $F$ the density of volumetric forces acting in the fluid and $\nabla$ the gradient operator.

The Reynolds-averaged approach is based on decomposing the velocity as $u_{j}=\bar{u}_{j}+u^{\prime}{ }_{j}$ where $\bar{u}_{j}$ is the average velocity vector and $u_{j}^{\prime}$ the velocity vector fluctuation. The average continuity and momentum equation for steady state incompressible flow are given by:

$$
\begin{aligned}
& \rho \nabla \cdot(\bar{U})=0 \\
& \rho(\bar{U} \cdot \nabla) \bar{U}=\nabla\left[-P+\mu\left(\nabla \bar{U}+(\nabla \bar{U})^{t}\right]-\rho \bar{u}^{\prime}\left(\nabla \bar{u}^{\prime}\right)+F\right.
\end{aligned}
$$

Equation (4) has the same form of the Navier-Stokes equation (2), but now it has an additional term, the turbulent Reynolds stress term, $-\rho \bar{u}^{\prime}\left(\nabla \bar{u}^{\prime}\right)$ representing the influence of the fluctuation on the average flow. In order to close Eqs. (3) and (4), the turbulent Reynolds stresses must be determined. These can be directly modeled through the Reynolds Stress Models (RSM) which involves calculation of the individual Reynolds stresses by using differential transport equations.

Another closure procedure is provided by the K- $\varepsilon$ turbulence model.

\subsection{The $K-\varepsilon$ turbulence model}

An extended K- $\varepsilon$ turbulence model is used here. It contains the standard two transport equations as proposed by Launder and Spalding and an improved transport equation for the rate of dissipation of the turbulent kinetic energy [8]. This model was found to give more effective response to the energy production rate than does the standard K- $\varepsilon$ turbulence model. The model equations write:

$$
\begin{aligned}
& \rho(\bar{U} . \nabla) K=\nabla\left[\left(\mu+\frac{\mu_{t}}{\sigma_{k}}\right) \nabla K\right]+P_{k}-\rho \varepsilon \\
& \rho(\bar{U} . \nabla) \varepsilon=\nabla\left[\left(\mu+\frac{\mu_{t}}{\sigma_{\varepsilon}}\right) \nabla \varepsilon\right]+C_{\varepsilon 1} \frac{\varepsilon}{K} P_{K}-C_{\varepsilon 2} \rho \frac{\varepsilon^{2}}{K} \\
& \mu_{t}=\rho C_{\mu} \frac{K^{2}}{\varepsilon} \\
& P_{K}=\mu_{t}\left[\nabla \bar{U}+(\nabla \bar{U})^{t}\right] \nabla \bar{U}
\end{aligned}
$$

The adaptation of the standard model constants which is applicable to flows with high Reynolds numbers is given in table 1.

Table 1. Values of the K- $-\varepsilon$ model constants

\begin{tabular}{ccccc}
\hline$C_{\mu}$ & $C_{\varepsilon 1}$ & $C_{\varepsilon 2}$ & $\sigma_{k}$ & $\sigma_{\varepsilon}$ \\
\hline 0.09 & 1.44 & 1.92 & 1 & 1.3 \\
\hline
\end{tabular}




\subsection{Boundary layer condition at the ground}

For a turbulent shear flow which is steady, the meanvelocity profile in a small region near the surface is described by a relationship called the wall law:

$\frac{u_{t}}{u^{*}}=\frac{1}{\kappa} \ln \left(\frac{z}{z_{0}}\right)+B$

where $u^{*}$ is the friction velocity, $\kappa$ is the von Karman constant $(\kappa=0.42), B$ is an empirical constant related to the thickness of the viscous sublayer $(B=5.2$ in a boundary layer over a smooth flat plane; for rough walls smaller values of $B$ are obtained). The constant $z_{0}$ called roughness is the frontier between the logarithmic zone and the inner K- $\varepsilon$ zone.

The determiantion of $z_{0}$ is considered usually through combining the logarithmic wall function as given in Eq. (10) and the numerical integration approach used. Denoting $u_{t}, u_{n}$ as respectively the tangential and normal velocity components, and $\partial u_{t} / \partial n$ the normal derivative of the tangential velocity, the wall law writes

$u_{n}=0$

$\frac{u_{t}}{\sqrt{\frac{\partial u_{t}}{\partial n}}}=\sqrt{\frac{\eta}{\rho}} \frac{1}{\kappa} \ln \left(\frac{z_{0}}{\sqrt{\frac{\partial u_{t}}{\partial n}}} \sqrt{\frac{\rho}{\eta}}\right)+2.4 \sqrt{\frac{\eta}{\rho}}$

with the following implicit condition to be satisfied on $z_{0}$

$$
20 \sqrt{\frac{\eta}{\rho}}<\frac{z_{0}}{\sqrt{\frac{\partial u_{t}}{\partial n}}}<100 \sqrt{\frac{\eta}{\rho}}
$$

\subsection{Boundary conditions}

Table 2 summarizes all the boundary conditions considered here.

Table 2: Considered boundary conditions

\begin{tabular}{|c|c|c|}
\hline & Parameter & Boundary condition \\
\hline The wall law & $\begin{array}{l}\text { height } \\
\text { roughness } z_{0} \\
{[\mathrm{~m}]}\end{array}$ & $\frac{u_{t}}{u_{*}}=\frac{1}{\kappa} \ln \left(\frac{z}{z_{0}}\right)+B$ \\
\hline Input section & $\begin{array}{l}\text { Logarithmic } \\
\text { velocity } \\
\text { profile }[\mathrm{m} / \mathrm{s}]\end{array}$ & $\begin{array}{l}u_{x}=u \\
u_{y}=0\end{array}$ \\
\hline $\begin{array}{l}\text { Upper edge of } \\
\text { the domain }\end{array}$ & $\begin{array}{l}\text { Uniform } \\
\text { velocity }[\mathrm{m} / \mathrm{s}]\end{array}$ & $u=u_{\text {uniform }}$ \\
\hline Output section & $\begin{array}{l}\text { Pressure } \\
{[\mathrm{atm}]}\end{array}$ & $P=P_{a t m}$ \\
\hline
\end{tabular}

\subsection{Mesh used}

For un unbounded domain, the primary difficulty in using CFD at a practical level is the uncertainty of creating a frontier and an initial mesh that is susceptible to produce a sufficiently accurate solution for the actual flow problem. Here, a parametric study has been performed in order to find the adequate domain and mesh size.

Let's consider the problem of a flat ground with an obstacle having the form of a half-ellipse: $125 \mathrm{~m}$ of great axis and $50 \mathrm{~m}$ of small axis. The air flowing on this ground has density $\rho=1.225 \mathrm{~kg} / \mathrm{m}^{3}$ and and dynamic viscosity $\eta=1.7894 \times 10^{-5} \mathrm{~kg} / \mathrm{m} / \mathrm{s}$.

The domain geometry that has yielded convergence is presented in Figure 1. It is limited by a rectangle having height $h=180 \mathrm{~m}$ and width $\ell=280 \mathrm{~m}$. This figure shows the mesh of the first model that corresponds to the case of a flat gournd with an obstacle. The same mesh size was used for the problem of flat ground without obstacle, and in both cases, the finite element used has triangular geometry and linear dinterpolation function.

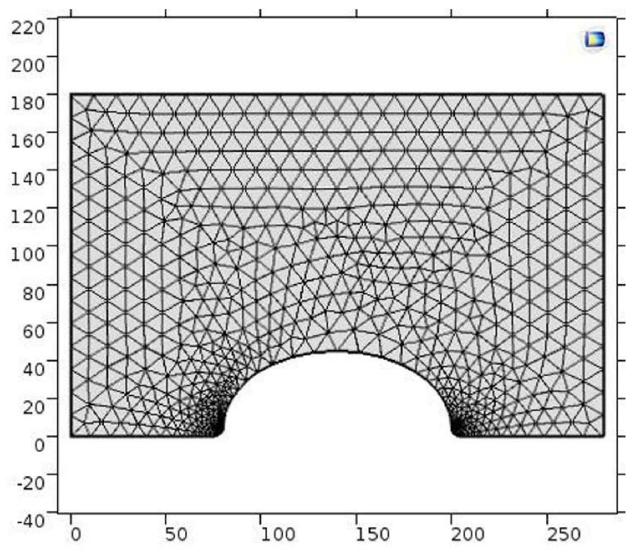

Fig 1. Mesh of the flat ground with obstacle.

\section{Results and discussion}

Taking an input air flow at the left boundary of the fluid domain that has the asymptotic magnitude of velocity $u_{\infty}=18.5 \mathrm{~m} / \mathrm{s}$, simulations have been performed according to section 2 indications. An iterative procedure based on the condition of obtaining an invariable solution with regards to the frontier location and mesh size has been applied.

Figure 2 gives the obtained isovalues of the air speed magnitude for the case of ground with an elliptic like obstacle.

Figure 3 gives the results in terms of isovalues of air speed magnitude for the problem of flat ground without obstacle.

Figure 4 gives the air speed magnitude as function of the location on the cut-lines that are shown on figures 3 and 4 as dashed lines. 


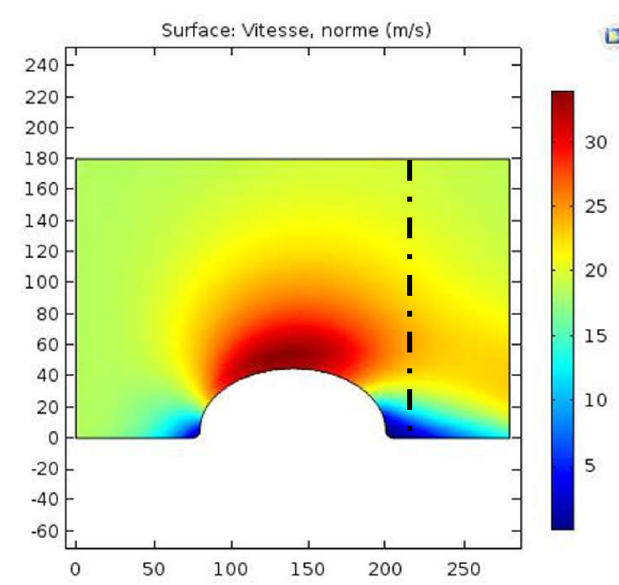

Fig.2. Isovalues of the air speed magnitude for the ground with an obstacle, the dashed line defines a cut-line.

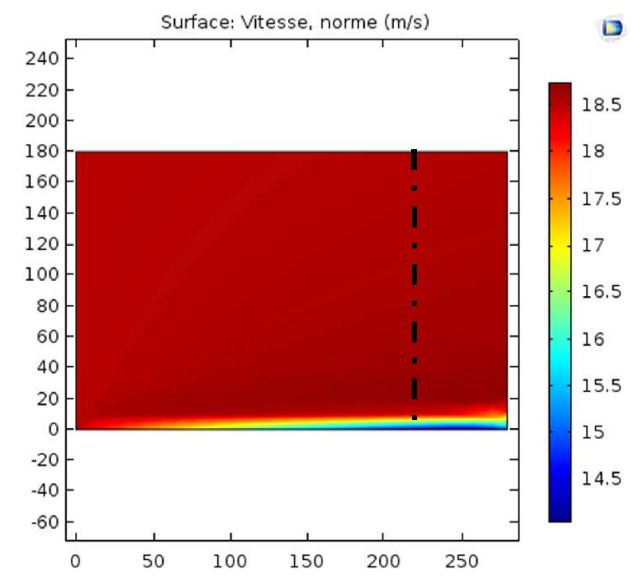

Fig.3. Isovalues of the air speed magnitude for the flat ground without obstacle, the dashed line defines a cut line.

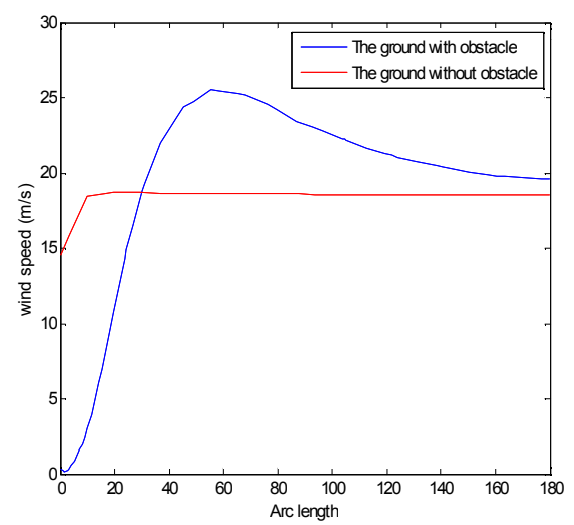

Fig.4. Air speed magnitude as function of the location on a cutline; comparison between the ground with and without obstacle

From figure 3, one can see that air flow undergoes and acceleration due the obstacle effect. The air velocity jumpt from as the air velocity increases up to $65 \mathrm{~m} / \mathrm{s}$.

Figure 5 shows that the air flow velocity is largely affected by the obstacle. It is reduced behind the obstacle and amplified above the obstacle.
The obtained results make it possible also to find the shear stress and turbulence intensity which can be used to make accurate estimation of wind energy as function of the wind turbine location.

\section{Conclusions}

The local circular motion as induced by the existence of an obstacle having the form of an ellipse has been analyzed under stationary air flow assumption. Computational fluid dynamics based on solution of the turbulent air flow equations that consist of the NavierStokes equations coupled to the K- $\varepsilon$ turbulence model was performed. The boundary conditions at the ground surface were applied by using an improved wall law. COMSOL software package was used. Accuracy of the model was assessed as function of both the location of the boundary fixing the truncated computational domain and the mesh size. The obtained results for the ground with an obstacle have shown significant discrepancies in comparison with the case of a flat ground without obstacle. The presence of a hill modifies drastically the air flow pattern and influnces hence to a large extent the extractable wind energy. This influence can be determined according to the actual obstacle geometry and location of the wind turbine.

\section{References}

1. P. Bahattacharya, wind energy management 1, InTech, (2011). DOI: 10.5772/1530.

2. A.D.Young, Boundary layers, BSP Professional Books, Oxford, 180-182 (1989).

3. J.D. Anderson, Fundamentals of aerodynamics, $4^{\text {th }}$ Edition McGraw-Hill, (2007).

4. Y.A. Cengel, Heat Transfer: A Practical Approach, $2^{\text {nd }}$ Edition McGraw-Hill, (2007).

5. J.H. Ferziger, M. Peric, Computational Methods for Fluid Dynamics, $3^{\text {rd }}$ Edition, Springer third, (2002). DOI: 10.1007/978-3-642-56026-2

6. J. Sumner, C.S. Watters, C. Masson. CFD in Wind Energy: The Virtual, Multiscale Wind Tunnel 3, 9891013 (2010). DOI: 10.3390/en3050989.

7. Introduction to COMSOL Multiphysics, version 5.1COMSOL, Sweden, (2015)

8. B.E. Launder, D.B. Spalding, The numerical computation of turbulent flows, Computer Methods in Applied Mechanics and Engineering 3, 269-289 (1974). 